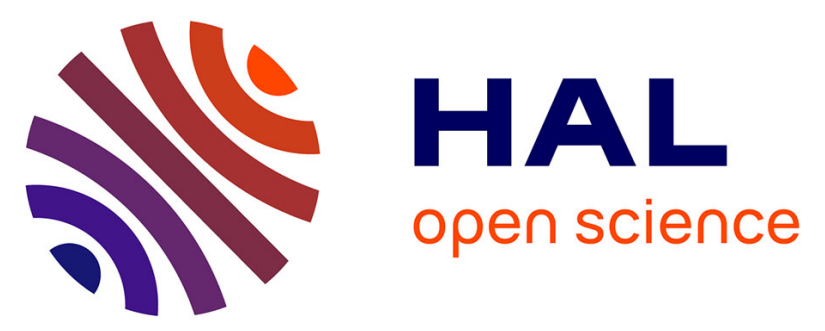

\title{
Control of adiabatic light transfer in coupled waveguides with longitudinally varying detuning
}

Hassan Oukraou, Laura Vittadello, Virginie Coda, Charles Ciret, Massimo Alonzo, Andon A. Rangelov, Nikolay V. Vitanov, Germano Montemezzani

\section{- To cite this version:}

Hassan Oukraou, Laura Vittadello, Virginie Coda, Charles Ciret, Massimo Alonzo, et al.. Control of adiabatic light transfer in coupled waveguides with longitudinally varying detuning. Physical Review A : Atomic, molecular, and optical physics [1990-2015], 2017, 95 (2), pp.023811(1-7). 10.1103/PhysRevA.95.023811 . hal-01499470

\section{HAL Id: hal-01499470 \\ https://hal-centralesupelec.archives-ouvertes.fr/hal-01499470}

Submitted on 7 May 2021

HAL is a multi-disciplinary open access archive for the deposit and dissemination of scientific research documents, whether they are published or not. The documents may come from teaching and research institutions in France or abroad, or from public or private research centers.
L'archive ouverte pluridisciplinaire HAL, est destinée au dépôt et à la diffusion de documents scientifiques de niveau recherche, publiés ou non, émanant des établissements d'enseignement et de recherche français ou étrangers, des laboratoires publics ou privés. 


\title{
Control of adiabatic light transfer in coupled waveguides with longitudinally varying detuning
}

\author{
Hassan Oukraou, ${ }^{1,2, *}$ Laura Vittadello, ${ }^{1,3}$ Virginie Coda, ${ }^{1,2}$ Charles Ciret, ${ }^{1,4}$ Massimo \\ Alonzo, ${ }^{1,5}$ Andon A. Rangelov, ${ }^{6}$ Nikolay V. Vitanov, ${ }^{6}$ and Germano Montemezzani ${ }^{1,2}$ \\ ${ }^{1}$ Université de Lorraine, LMOPS, 2, rue E. Belin, 57070 Metz, France \\ ${ }^{2}$ CentraleSupélec, LMOPS, 2, rue E. Belin, 57070 Metz, France \\ ${ }^{3}$ Universitá di Padova, Dipartimento di Fisica e Astronomia G. Galilei, Via Marzolo 8, 35131 Padova, Italy \\ ${ }^{4}$ OPERA-Photonique, Université libre de Bruxelles (ULB), \\ 50 av. F.D. Roosevelt, CP194/5, 1050 Bruxelles, Belgium \\ ${ }^{5}$ Ultrafast Photonics Laboratory, Dipartimento di Energetica, \\ Sapienza Universitá di Roma and CNISM, 00161 Roma, Italy \\ ${ }^{6}$ Department of Physics, Sofia University, James Bourchier 5 blvd, 1164 Sofia, Bulgaria
}

\begin{abstract}
We study adiabatic light transfer in systems of two coupled waveguides with spatially varying detuning of the propagation constants, providing an analogy to the quantum phenomena of Rapid Adiabatic Passage (RAP) and two-state Stimulated Raman Adiabatic Passage (two-state STIRAP). Experimental demonstration using a photo-induction technique confirms the robust and broadband character of the structures that act as broadband directional couplers and broadband beam-splitters, respectively.
\end{abstract}

\section{INTRODUCTION}

The formal analogy between some quantum systems and classical photonic systems has motivated intensive research in recent years, both from a fundamental and application-oriented point of view $[1,2]$. In this context a strong attention was given to the coupling and passage of light between waveguides, which behaves similarly to the population transfer in quantum systems driven by an appropriate external field. Several studies in waveguides were inspired by the Stimulated Raman Adiabatic Passage process (STIRAP) [3-5], which represents a very effective and robust way to coherently control the population of quantum states by means of a proper adiabatic temporal evolution of the coupling hamiltonian. In this way the system population can be effectively driven from an initial state to a final target state. The first proposals $[6,7]$ and the first experimental demonstration [8] of a STIRAP-like process in waveguides have stimulated several studies on adiabatic light passage in waveguides by slight modifications of this concept. These include theoretical and experimental studies related to the fractional STIRAP process [9], multi-state STIRAP [10-12], beamsplitting $[9,13-16]$, adiabatic mode conversion $[11,17]$ the role of nonlinear effects [18], or the use of such waveguide structures for photonic quantum gate operations [16]. Generally these approaches profit from the high robustness of the adiabatic process, leading for instance to a broadband behavior of the light spatial adiabatic passage process. However, since the necessary adiabatic condition is better fulfilled at longer than at shorter wavelengths [12], the use of such systems for spectral low-pass or high-pass filtering was also proposed [19, 20].

\footnotetext{
* hassan.oukraou@centralesupelec.fr
}

Previous studies principally rely on an adiabatic spatial evolution of the coupling constant $C$ between neighboring waveguides. As follows from the coupled-mode theory [21], the latter is mainly connected to the distance between the evanescently coupled waveguides. Its counterpart in atomic physics is a temporal evolving Rabi frequency $\Delta \Omega$ coupling the quantum states. In the case of atomic physics the atom-laser detuning $\Delta \omega$ provides a useful and versatile parameter for the adiabatic control of the system evolution. This detuning is given by a temporal dependent mismatch of the frequency of the driving field from the transition frequency. The corresponding parameter in waveguides is represented by spatial evolution of a detuning $\Delta \beta$ of the propagation constants in the individual waveguides. Therefore, it is expected that also for waveguides the detuning parameter shall increase the possibilities for controlling the adiabatic evolution of the propagating light fields. Even though the interest of using tapered waveguides was recognized early for the optimization of various components [22-24], little attention was given to the combination with a spatially evolving coupling coefficient.

In the present work we address this issue in relation with existing quantum phenomena. We consider two coupled waveguides with spatially varying coupling coefficients and we study theoretically and experimentally the effect of the detuning $\Delta \beta(z)$ (associated to a longitudinal variation of the index contrast) on the adiabatic light transfer between them. We choose two concrete examples. The first mimics the Rapid Adiabatic Passage (RAP) process [4] in quantum physics for which the detuning crosses zero while the coupling pulse is applied, which leads to a robust and rapid way to adiabatically invert a two-state system. Such a population transfer by adiabatic passage via a level crossing was initially implemented in nuclear magnetic resonance [25]. Laser-driven adiabatic passage in atoms and molecules was proposed 
by Treacy [26] and demonstrated first in the infrared by Stark-shifting the transition frequency [27], or by sweeping the laser frequency through resonance [28]. In the 80 s, adiabatic passage was observed also in the nearinfrared [29] and with visible light [30]. Further details can be found in Refs. [4, 31,32]. The second example mimics the so-called two-state STIRAP process [33, 34], which leads finally to an equal coherent superposition of the two states. Two-state STIRAP was experimentally demonstrated by Yamazaki et al. [35] with a trapped ${ }^{40} \mathrm{Ca}^{+}$ion. In the case of waveguides the RAP process implements an achromatic directional coupler, while the two-state STIRAP process implements a broadband oneto-two beam splitter. The experiments are performed with the help of reconfigurable photoinduced waveguides recorded through the photorefractive effect using the lateral illumination technique $[12,36,37]$. Two probing wavelengths more than $200 \mathrm{~nm}$ apart are used. Section II gives a general framework for the studies, section III addresses the case of the RAP-like process, while section IV treats the case of two-state STIRAP. The experimental results are in good agreement with the theoretical expectations and confirm the crucial role of the waveguide detuning.

\section{GENERAL FRAMEWORK}

We consider two neighboring waveguides with different longitudinal propagation constants $\beta_{1}(z)$ and $\beta_{2}(z)$ for their fundamental mode. In paraxial approximation, the propagation of a monochromatic light wave in such a structure can be analyzed in the framework of the coupled mode theory (CMT)[21] which treats the problem in a discrete way by involving the evanescent coupling between the waveguides. The corresponding evolution of the waves amplitudes can then be described by two coupled differential equations, which, similarly as in $[6,37,38]$, read as follows in the present case

$$
i \frac{d}{d z}\left[\begin{array}{l}
a_{1}(z) \\
a_{2}(z)
\end{array}\right]=\left[\begin{array}{cc}
-\Delta \beta(z) / 2 & C(z) \\
C(z) & \Delta \beta(z) / 2
\end{array}\right]\left[\begin{array}{l}
a_{1}(z) \\
a_{2}(z)
\end{array}\right] .
$$

Here the components of $\mathbf{A}(z)=\left[a_{1}(z), a_{2}(z)\right]^{T}$ are, besides for an unimportant phase, proportional to the amplitudes of the fundamental modes in the two waveguides. We call $\mathbf{H}(z)$ the coupling matrix in the above equation, which takes the role of the Hamiltonian and describes the interaction between the waveguide modes. It depends on the detuning parameter $\Delta \beta(z)=\beta_{2}(z)-\beta_{1}(z)$ as well as on the space-dependent coupling constant $C(z)$ between the two waveguides. Note that the latter corresponds to the geometrical average of the values for the coupling from waveguide 1 to 2 and from waveguide 2 to 1 [37], $C(z)=\sqrt{C_{12}(z) C_{21}(z)}$, which can be different due to the local asymmetry. Note also that the driving quantity for $C(z)$ is the lateral distance $d(z)$ between the waveguides. Even though an exact analytic expression cannot be given in our case, $C(z)$ decreases nearly exponentially with $d(z)$.

By substituting the space coordinate $z$ for the time coordinate $t$ in the Schrödinger-type equation (1), the two-waveguides system is fully equivalent to a coupled quantum two-level system, which population amplitude dynamics is described by the same equation within the rotating wave approximation $[4,39]$. The role of the coupling constant $C(z)$ is then taken by the Rabi frequency $\Omega(t)$ and the waveguide detuning $\Delta \beta(z)$ is equivalent to the atom-laser detuning $\Delta \omega(t)$.

Equation (1) is expressed in the natural basis formed by the modes of the two waveguides, called diabatic basis. However, we can rewrite it in another basis, called adiabatic basis, where the basis vectors are the local (instantaneous) eigenvectors of the space-varying Hamiltonian. This leads to

$$
i \frac{d}{d z}\left[\begin{array}{l}
b_{1}(z) \\
b_{2}(z)
\end{array}\right]=\left[\begin{array}{cc}
-\epsilon(z) & -i d \theta / d z \\
i d \theta / d z & \epsilon(z)
\end{array}\right]\left[\begin{array}{l}
b_{1}(z) \\
b_{2}(z)
\end{array}\right],
$$

where the quantities $\epsilon$ and $\theta$ are given by

$$
\epsilon(z)=\left(C^{2}(z)+(\Delta \beta / 2)^{2}\right)^{1 / 2},
$$

and

$$
\theta(z)=\frac{1}{2} \arctan (2 C(z) / \Delta \beta(z))
$$

The vector $\mathbf{B}(z)=\left[b_{1}(z), b_{2}(z)\right]^{T}$ contains the modes amplitudes in the adiabatic basis. The amplitudes in the adiabatic and diabatic basis are connected by a space-dependent rotation operator such that $\mathbf{A}(z)=$ $\mathbf{R}(\theta(z)) \mathbf{B}(z)$, with

$$
\mathbf{R}(\theta(z))=\left[\begin{array}{cc}
\cos \theta(z) & \sin \theta(z) \\
-\sin \theta(z) & \cos \theta(z)
\end{array}\right]
$$

For an adiabatic evolution, the amplitudes $C(z)$ and $\Delta \beta(z)$ should vary slowly so that $\theta(z)$ evolves smoothly in order to ensure that the adiabatic state vector $\mathbf{B}(z)$ remains fixed in the adiabatic basis. To do so, the following condition should be fulfilled [4]

$$
\frac{1}{2}\left|\frac{\partial C}{\partial z} \Delta \beta-C \frac{\partial \Delta \beta}{\partial z}\right| \ll\left(C^{2}+\left(\frac{\Delta \beta}{2}\right)^{2}\right)^{3 / 2} .
$$

When the adiabatic condition holds, the state vector $\mathbf{B}(z)$ remains fixed in the space-varying basis formed by the adiabatic states. In particular, if the state vector $\mathbf{B}(z)$ coincides with a single adiabatic state at some distance $z$, then it will remain in that adiabatic state as long as the evolution is adiabatic, the state vector $\mathbf{B}(z)$ will adiabatically follow this state.

The two eingenstates (the adiabatic states) of the rotation matrix (5) are

$$
\begin{aligned}
& \mathbf{B}_{-}(z)=\mathbf{A}_{1} \cos \theta(z)-\mathbf{A}_{2} \sin \theta(z), \\
& \mathbf{B}_{+}(z)=\mathbf{A}_{1} \sin \theta(z)+\mathbf{A}_{2} \cos \theta(z),
\end{aligned}
$$



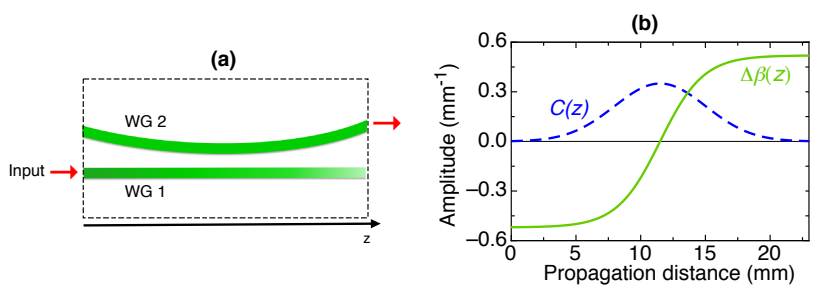

(c)

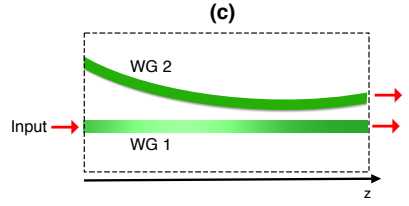

(d)

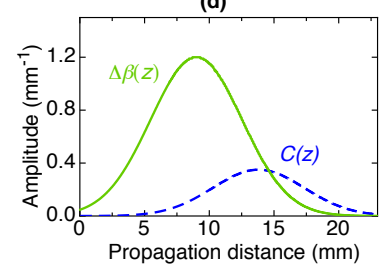

FIG. 1. (a) Schematics of the waveguide structure providing an optical analogy to the RAP process and, (b), corresponding longitudinal evolution of the coupling constant $C(z)$ and of the detuning $\Delta \beta(z)$. (c) Schematics of the waveguide structure providing an optical analogy to the two-state STIRAP process and, (d), corresponding evolution of $C(z)$ and $\Delta \beta(z)$ (case A). Note that on the schemes (a) and (c) the longitudinal and transverse dimensions are not in scale.

where $\mathbf{A}_{1}$ and $\mathbf{A}_{2}$ are the two diabatic states of the system. In general, the state vector $\mathbf{B}(z)$ is aligned with an initial state at the beginning of the propagation and aligned with a specified target state at the end of the propagation. Under appropriate conditions, this alignement can be provided by a single adiabatic state or by a superposition of the adiabatic states. In the first situation, this state is called an adiabatic transfer state.

In the next sections we will apply and verify the above general behavior to the specific cases of the RAP-like and two-state STIRAP-like light transfer. The waveguide structures being studied are shown schematically on Fig. 1. They are composed of two planar waveguides where the light is confined only in the plane of the drawing. One of the waveguide is straight (WG1) while the other is weakly curved (WG2), so that the distance separating them and the coupling constant $C$ evolves with $z$. The propagation constant $\beta_{1}$ of WG1 changes with $z$, which is achieved in our case by means of a variation of its refractive index contrast. The propagation constant $\beta_{2}$ is left constant.

\section{RAP-LIKE LIGHT TRANSFER}

The waveguide structure used to simulate the RAP process is shown schematically in Fig. 1(a). The corresponding evolution of the parameters $C(z)$ and $\Delta \beta(z)$ are shown in Fig. 1(b). These parameters mimic the experimental situation discussed below. For the RAP process to occur, it is essential that the detuning should cross zero during the evolution, which is the case for the function $\Delta \beta(z)$ in Fig. 1(b), that follows a tanh function with its zero corresponding to the maximum of $C(z)$ (nearest distance between the waveguides). At early and

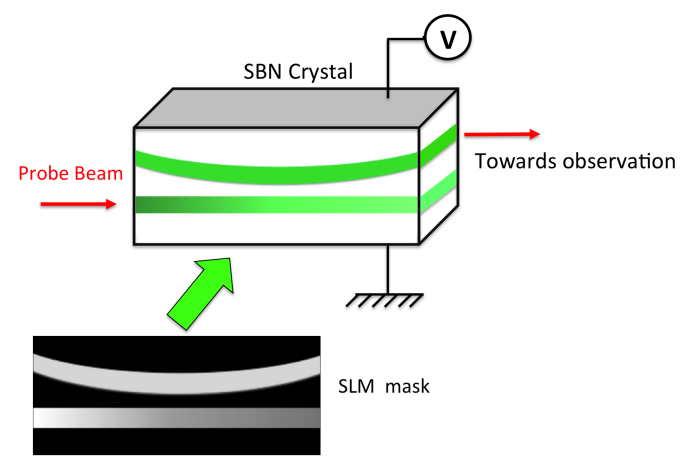

FIG. 2. Schematics of the technique to create the reconfigurable waveguide structures. A control beam at the wavelength $\lambda=532 \mathrm{~nm}$ carries the structure of the waveguides. The latter is shaped by a spatial light modulator (SLM) and imaged to the surface of the SBN crystal, to which an electric field is applied along its polar axis. The probe beam (alternatively at $\lambda=633 \mathrm{~nm}$ or $\lambda=850 \mathrm{~nm}$ ) propagates through the resulting waveguide structures to test their functionalities.

late distances the magnitude $|\Delta \beta(z)|$ is much larger than $C(z)$, while the contrary is true near the zero-crossing point. Since the mixing angle $\theta(z)$ depends strongly on the ratio $C / \Delta \beta$ (see Eq. (4)), $\theta(z)$ will evolve during the propagation. Initially $(z=0)$, this ratio tends to 0 and $2 \theta$ is nearly equal to $\pi$, so that $\theta \approx \pi / 2$. With inscreasing $z, C(z)$ and $\Delta \beta(z)$ increase $(\Delta \beta(z)$ approaches zero from the negative side), which leads to a decrease of $\theta(z)$. At half distance, $\Delta \beta(z)$ vanishes and $C(z)$ reaches its maximum, so that $2 \theta=\pi / 2$ and $\theta=\pi / 4$. In the following $\Delta \beta(z)$ continues to increase and becomes again much larger than $C(z)$, which is decreasing, so that $\theta$ tends to zero at large distances. Consequently, starting initially from state $A_{1}$ (all light in WG1), the system follows adiabatically the adiabatic state $B_{+}(z)$ of Eq. (7b) and eventually ends up in state $A_{2}$ (all light in WG2). This implies that a complete light transfer should take place. This adiabatic process is intrinsically robust and is expected to take place over a wide range of the design parameters and of the propagating wavelengths for a given design, which means that the behavior should be highly achromatic.

We have verified the above expectations by using photoinduced dynamic waveguides recorded by a properly structured lateral control illumination on a $\mathrm{Sr}_{x} \mathrm{Ba}_{1-x} \mathrm{Nb}_{2} \mathrm{O}_{6}$ (SBN) crystal with $x=0.61$. A highly simplified conceptual scheme of this method is shown in Fig. 2. Essentially, the combination of a local illumination by the control beam and of the electric field applied to the photorefractive SBN crystal leads to a local modification of the refractive index landscape of the crystals. This modification is such that maxima of the refractive index are found at the positions of maximum illumina- 
tion, leading therefore to a guiding waveguide structure. Variations of the index contrast (and of the propagation constant) can be achieved by varying locally the intensity (grey level) of the control light being directed from the spatial light modulator (SLM) to the crystal. Since the recording and erasure of the structures takes only few tens of seconds in SBN, the technique provides the advantage of being able to produce reconfigurable structures in a unique crystal. Moreover, the dynamics of the waveguide recording process can be exploited to characterize the index contrast interferometrically. Since the technique was used earlier $[12,36,40]$, we refer to the corresponding works for additional details. Note that, even though for the sake of simplicity we use here waveguides confined in one transverse dimension, the confinement in both transverse directions can be achieved too [36].

The design of the waveguide configuration used to demonstrate the RAP-like effect is such that both $C(z)$ and $\Delta \beta(z)$ have a smooth evolution to satisfy at best the adiabaticity criterion within the limits permitted by the physics of our experimental approach. The nominal $z$-dependences corresponding to Fig. 1(b) are $C(z)=C_{\max } \exp \left[-\left(\left(z-z_{0}\right) / \sigma_{0}\right)^{2}\right]$ and $\Delta \beta(z)=$ $\Delta \beta_{\max } \tanh \left[2 \pi\left(z-z_{0}\right) / L\right]$, where $C_{\max }$ is the maximum of the coupling constant reached at half the propagation distance $z_{0}=11.5 \mathrm{~mm}, \sigma_{0}=5 \mathrm{~mm}$ is the $1 / \mathrm{e}$ half-width of the gaussian coupling-constant pulse, $\Delta \beta_{\max }$ is the maximum of detuning, and $L=23 \mathrm{~mm}$ is the full propagation distance in the crystal. The parameters $C_{\max }$ and $\Delta \beta_{\max }$ depend not only on the design of the waveguide but also on the probe wavelength. Their values were estimated by carrying out preliminary experiments in non-modulated waveguides. While the observation of the coupling of waves in parallel waveguides leads to an estimation of the coupling constant, the estimation of $\Delta \beta$ requires to perform interferometric measurement during the formation process of the waveguides. For our experimental conditions we estimate the parameters within a precision of roughly $15 \%, C_{\max }=0.35 \pm 0.05 \mathrm{~mm}^{-1}$ and $\Delta \beta_{\max }=0.52 \pm 0.08 \mathrm{~mm}^{-1}$ for the probe wavelength of $633 \mathrm{~nm}$, as well as $C_{\max }=0.49 \pm 0.07 \mathrm{~mm}^{-1}$ and $\Delta \beta_{\max }=0.32 \pm 0.05 \mathrm{~mm}^{-1}$ for the probing at $850 \mathrm{~nm}$. With the above parameters the expected evolution of the light intensity in the two-waveguide structure of Fig. $1(\mathrm{a})+(\mathrm{b})$ can be obtained from Eq. (1) and is shown in Figs. 3(a) and 3(b), for the wavelengths 633 and 850 $\mathrm{nm}$, respectively. In both cases at the output of the structure one expects essentially a switch from WG1 to WG2. The corresponding experimental results are shown on Fig. 3(c) and 3(d), which depict the intensity profile at the output of the crystal. The dashed blue lines give the output of the reference waveguide, that is WG1 in absence of WG2, while the solid red lines give the output when the RAP-like structure including the WG2 is produced. The experimental profiles show some slight asymmetry and irregularities associated mainly to experimental effects such as possible crystal imperfections and partial back-scattering of the recording light discussed

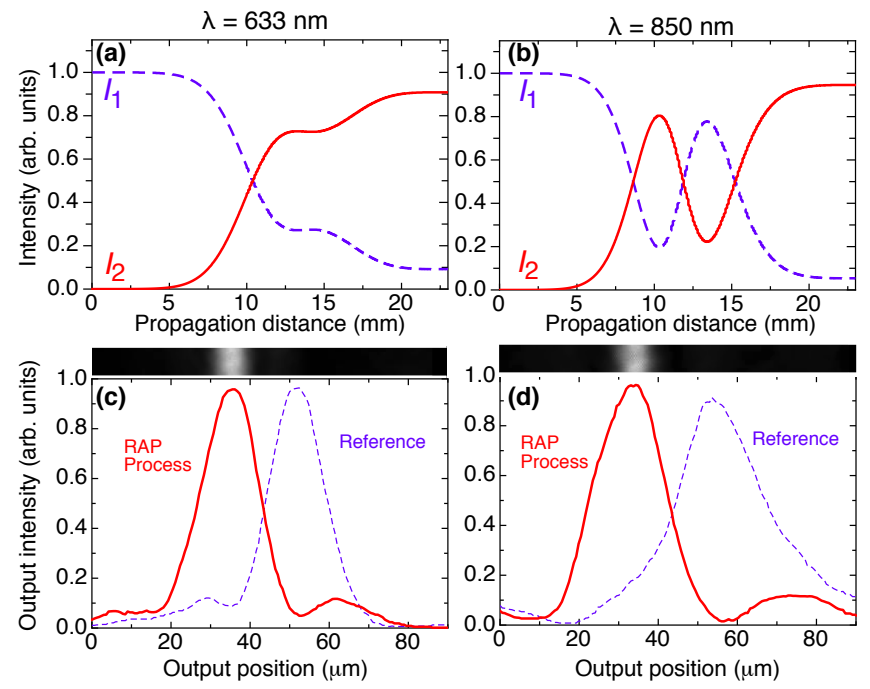

FIG. 3. Theoretical expectation and experimental verification of the RAP-like light transfer for the parameter given in the text. (a) Numerically calculated spatial evolution of the intensity in WG1 (blue dashed curve) and WG2 (solid red curve) for $\lambda=633 \mathrm{~nm}$. (b) Same for $\lambda=850 \mathrm{~nm}$. (c) Measured intensity output distribution with WG1 but in the absence of WG2 (reference, blue dashed line) and with the presence of both waveguides (solid red line). (d) Same for $\lambda=850 \mathrm{~nm}$ and the same structure.

in Ref. [41]. Nevertheless it can be recognized that for the RAP-like structure most of the light output essentially switches to WG2 for both wavelengths. This corresponds to the expectations and confirms the robustness of the approach.

It is worth verifying that the detuning is essential for the above RAP-like light transfer to occur. Figure 4 shows the expected and measured behavior for the same kind of two-waveguide structure leading to the results in Fig. 3, but in the absence of detuning $(\Delta \beta(z)=0)$. As seen in Fig. 4 in this case the RAP process does not take place and the results are not robust. An essentially complete return of the light to the straight waveguide is expected for $\lambda=633 \mathrm{~nm}$, while a strong transfer to the curved waveguide is expected for $\lambda=850 \mathrm{~nm}$ (see Fig. $4(\mathrm{a})$ and (b)). This is confirmed by the experimental results of Fig. 4(c) and 4(d), respectively. Therefore, such a structure essentially leads to a damped Rabi-like oscillation between the two waveguides, with very different output distributions for the two probing wavelengths.

Even though the experiments on the RAP-like light transfer of Fig. 3 give satisfactory results, the corresponding experimental parameters are still at the edge of the zone for which the adiabatic condition is fulfilled at best in the $\left(C_{\max }, \Delta \beta_{\max }\right)$-space. In order to illustrate this, we study the robustness with respect to these two parameters by plotting the expected intensity transferred to WG2 as a function of $C_{\max }$ and $\Delta \beta_{\max }$. Figure 5 (a) gives a contour plot of the theoretically expected transferred intensity as a function of these two parameters. Clearly the experimental parameters (blue circle for 

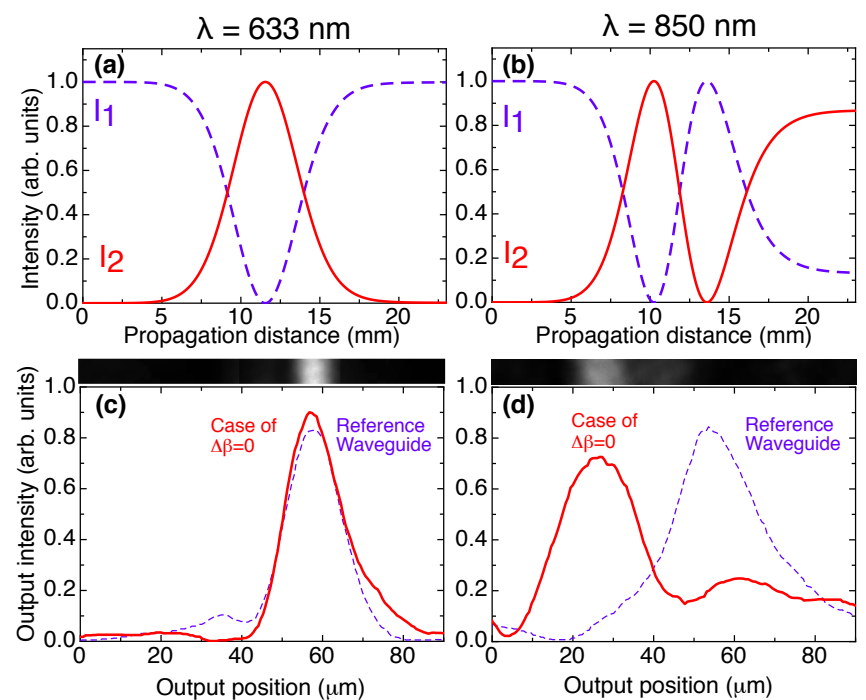

FIG. 4. Counter example to the RAP process in absence of the detuning $(\Delta \beta(z)=0)$. (a) Expected evolution of the intensity in WG1 (blue dashed curve) and WG2 (solid red curve) according to Eq. (1) for $\lambda=633 \mathrm{~nm}$. (b) Same for $\lambda=850 \mathrm{~nm}$. (c) Measured intensity output distribution with WG1 but in the absence of WG2 (reference, blue dashed line) and with the presence of both waveguides (solid red line). (d) Same for $\lambda=850 \mathrm{~nm}$ and the same structure.

$633 \mathrm{~nm}$ and green square for $850 \mathrm{~nm}$ ) are at the border of the red region of maximum adiabaticity. The counter example in absence of detuning corresponds to points on the abscissa of the diagram of Fig. 5(a). It is also worth noting that upon an increase of the wavelength, one gets a higher coupling constant but a lower detuning. This means that the increase of wavelength corresponds to moving transversally with respect to the direction leading to a maximum fulfillment of the adiabatic condition. The decrease in the detuning is due on one hand to the proportionality of $\beta$ on $1 / \lambda$ and, on the other hand, to a slight decrease of the electro-optic response and of the index contrast in the SBN material for longer wavelengths.

\section{TWO-STATE STIRAP-LIKE BEAM SPLITTING}

The concept of two-state STIRAP [33] relies on a mathematical analogy between the equations governing the population dynamics of a two-level system with a temporally varying detuned coupling field and a resonantly coupled chainwise-linked three-level system leading to the standard STIRAP process. As discussed above, the two-level system is described by a time-dependent Schrödinger equation of two complex variables. The latter can be recast as three coupled equations of three real variables $[39,42]$ in the form of the optical Bloch equation. This, on its turn, can be brought in a form equivalent to the Schrödinger equation applying to the standard STIRAP process in a three-level system. In this way,
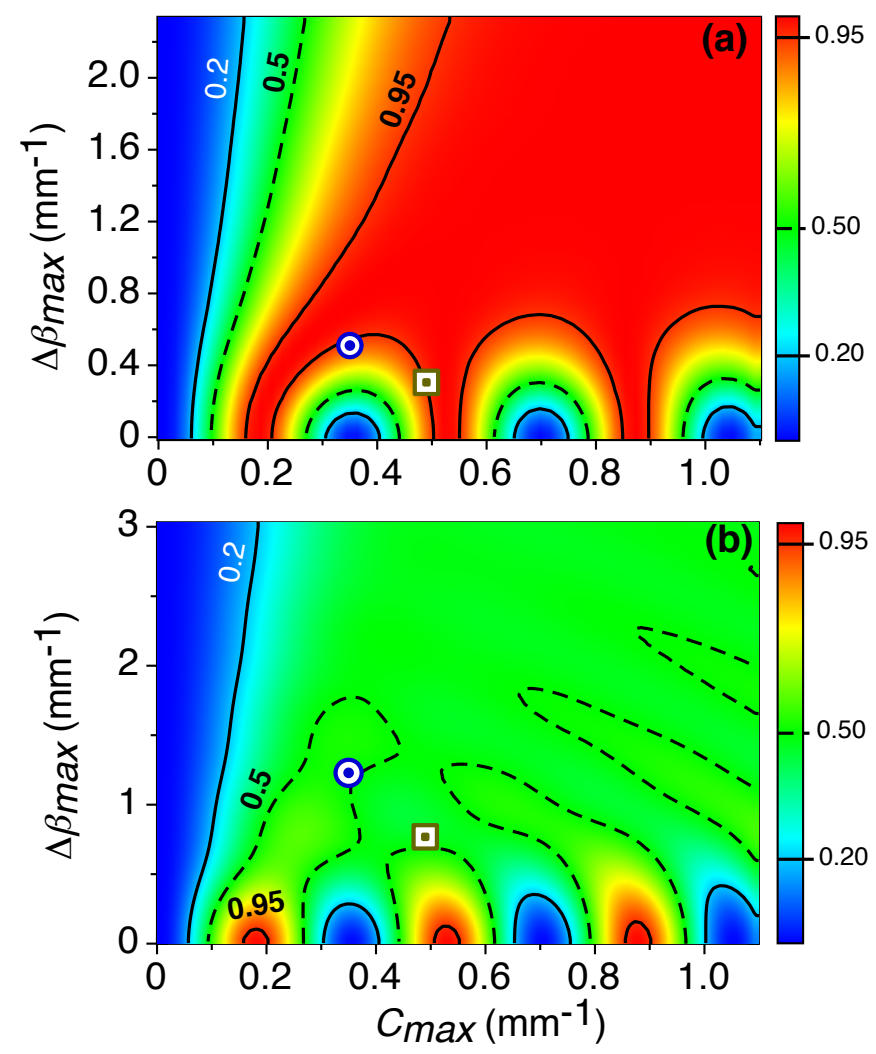

FIG. 5. (a) Landscape of the expected output intensity of WG2 for the RAP-process as calculated using coupled mode theory under variation of the parameters $C_{\max }$ and $\Delta \beta_{\max }$. The red area correspond to the region where the adiabatic condition is fulfilled the best and the light transfer is complete. The blue circle and the green square correspond to the experimental conditions of Fig. 3 for probing at $633 \mathrm{~nm}$ and $850 \mathrm{~nm}$, respectively. (b) Corresponding landscape for the two-state STIRAP process (case A) showing the relative intensity transferred from WG1 to WG2. The green area gives the region where the adiabatic condition is best fulfilled and the waveguide structure gives essentially a 50:50 beam splitter. The blue circle and the green square correspond to the experimental conditions of Fig. 7 for probing at $633 \mathrm{~nm}$ and $850 \mathrm{~nm}$, respectively.

knowledge about the STIRAP process can be directly inherited for the chirped two-state excitation [5, 33]. Ultimately the detuning of the two-level excitation can be identified with the Stokes pulse of a three-level STIRAP system (the Rabi-frequency pulse that couples the target state and the intermediate state), while the Rabifrequency of the two-level system is identified with the corresponding pump pulse of STIRAP, which couples the initial and the intermediate state.

For the analogy between the two-state STIRAP process and the coupling within a pair of detuned waveguides we can consider two distinct cases. The first one, Case $A$, is the one where the waveguide detuning $\Delta \beta(z)$ is spatially preceding the coupling constant pulse $C(z)$. This case is equivalent to a counter-intuitive sequence in the standard three-state STIRAP process $[3,5]$. It corre- 
sponds to the situation shown in Fig. 1(c) and Fig. 1(d). The second case, Case B, has the coupling preceding the detuning and would correspond to an intuitive order of the pulses for an atomic three-level system.

We start by discussing Case $A$ with the help of the general relationships given in Section II. Initially only $\Delta \beta(z)$ is present (see Fig. 1(d)) and its amplitude increases continuously, while $C(z)$ is negligibly small. Therefore the ratio $\Delta \beta(z) / C(z) \rightarrow+\infty$ and the mixing angle $\theta(z)$ in (4) is initially equal to zero, as shown in Fig. 6(a). With (7a) this implies that the state $\mathbf{B}_{-}$is initially aligned with $\mathbf{A}_{1}$. Subsequently, $\Delta \beta(z)$ increases towards its maximum value, as long as $C(z)$ is still small, $\mathbf{B}_{-}$deviated only little from the state $\mathbf{A}_{1}$. When $\Delta \beta(z)$ is behind its maximum and $C(z)$ reaches comparable values, the angle $\theta$ rises and the adiabatic transfer state $\mathbf{B}_{-}$is no longer aligned with $\mathbf{A}_{1}$ but becomes a superposition of $\mathbf{A}_{1}$ and $\mathbf{A}_{2}$. At the final stage, $\Delta \beta(L)$ tends to zero and $\Delta \beta(L) / C(L) \rightarrow 0$, so that $\theta(L)$ tends to $\pi / 4$. Consequently, the system shall tend to $\mathbf{B}_{-}(L)=\left(\mathbf{A}_{1}-\mathbf{A}_{2}\right) / \sqrt{2}$, which leads to an equal intensity of the light in the two waveguides, as shown in Fig. 6(c).
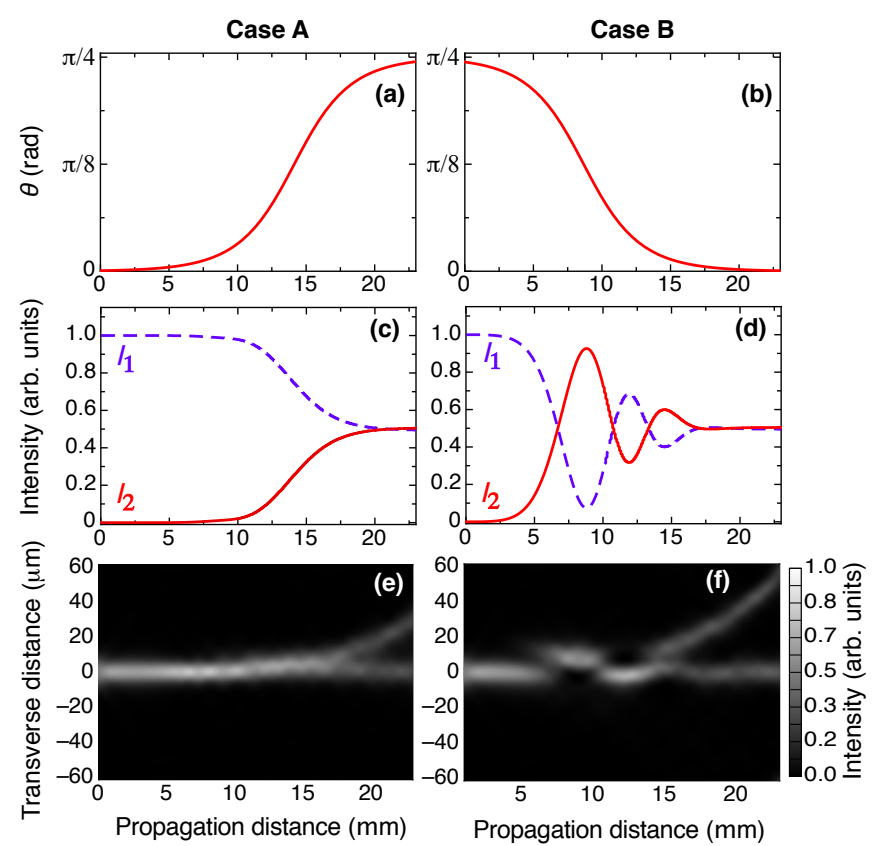

FIG. 6. Theoretical expectations for a two-state STIRAP-like waveguide structure modeled for propagation of light at the $633 \mathrm{~nm}$ wavelength. Panels (a), (c) and (e) correspond to case A, panels (b), (d) and (f) to case B. (a) and (b) show the evolution of the mixing angle $\theta(z)$ with the propagation distance $z$. (c) and (d) show the intensity evolution in WG1 (dashed blue line) and WG2 (solid red line) obtained from coupled mode theory. Panels (e) and (f) show the corresponding evolution of the light distribution obtained with the alternative beam propagation method (BPM). For both cases the system leads to an equal splitting of the input intensity among the two waveguides.

The Case $B$ corresponds to the situation where the light is injected in WG1 from the right side of the struc- ture in Fig. 1(c). In this case, the ratio $\Delta \beta(z) / C(z)$ goes from 0 to $+\infty$ and $\theta(z)$ (see figure $6(\mathrm{~b})$ ) from $\pi / 4$ to 0. Importantly, in this situation the initial state $\mathbf{A}_{1}$ (injection in WG1) does not correspond to one of the adiabatic states $\mathbf{B}_{-}$or $\mathbf{B}_{+}$, but to a superposition $\mathbf{B}^{*}$ of them given by

$$
\mathbf{B}^{*}(z)=\frac{1}{\sqrt{2}}\left(\mathbf{B}_{-}(z)+\mathbf{B}_{+}(z)\right) .
$$

Therefore, unlike for case A where only one adiabatic state is involved, here the system is expected to remain in the superposition state $\mathbf{B}^{*}$ as long as the system evolves adiabatically. Since at the end of the structure $\theta(L)=$ 0 , the system shall converge towards $\mathbf{B}^{*}(L)=\left(\mathbf{A}_{1}+\right.$ $\left.\mathbf{A}_{2}\right) / \sqrt{2}$, which is again an equal intensity distribution of the light in the two waveguides, as shown in Fig. 6(d). However, unlike for case A, the evolution towards this 50:50 splitting presents here some transient oscillations.

The curves shown in Fig. 6(a)-(d) are modeled according to the parameters used for the experiments given below and the following functions, $\Delta \beta(z)=$ $\Delta \beta_{\max } \exp \left[-\left(\left(z-z_{1}\right) / \sigma_{0}\right)^{2}\right]$ and $C(z)=C_{\max } \exp [-((z-$ $\left.\left.\left.z_{2}\right) / \sigma_{0}\right)^{2}\right]$, where $z_{1}=9 \mathrm{~mm}$ and $z_{2}=13.8 \mathrm{~mm}$ for case A, and $z_{1}=13.8 \mathrm{~mm}$ and $z_{2}=9 \mathrm{~mm}$ for case B. A common $1 / e^{2}$ half-width of $\sigma_{0}=5 \mathrm{~mm}$ is used. The values of $\Delta \beta_{\max }$ and $C_{\max }$ are those for the wavelength of $633 \mathrm{~nm}$, which are shown as a blue point on the landscape applying to the two-state STIRAP case in Fig. 5(b). These are $\Delta \beta_{\max }=1.2 \pm 0.18 \mathrm{~mm}^{-1}$, and $C_{\max }=0.35 \pm 0.05 \mathrm{~mm}^{-1}$. The corresponding values for the same structure but for the wavelength of $850 \mathrm{~nm}$ (green square in Fig. $5(\mathrm{~b})$ ) are $\Delta \beta_{\max }=0.73 \pm 0.11$ $\mathrm{mm}^{-1}$ and $C_{\max }=0.52 \pm 0.08 \mathrm{~mm}^{-1}$, where the errors correspond to the experimental conditions. Figure 6 also contains numerical calculations of the beam propagation in waveguide structures corresponding to case A and case B using the Beam Propagation Method (BPM) [43, 44]. This second method has been widely used to design optical waveguides and predict the light evolution in such structures. The design structure only takes as an input the form and the index profile of the waveguides and does not assume anything with respect to coupled wave theory. As can be seen in Figs. 6(e) and 6(f), the BPM calculations also correctly predict the smooth evolution for case $\mathrm{A}$, and the initial oscillations for case $\mathrm{B}$, in agreement with the curves in Figs. 6(c) and 6(d) obtained from coupled wave theory.

Therefore, the theory predicts that at the output of the waveguide structures that mimic two-state STIRAP one should obtain a similar splitting irrespective of case A or B, and irrespective of the wavelength (see Fig. 5(b)), provided that the system fulfills sufficiently well the adiabatic condition. Figure 7 gives the experimental output distributions for propagation in photoinduced waveguide structures corresponding to cases A and B at the probe wavelengths of 633 and $850 \mathrm{~nm}$. It is seen that the beam splitting is satisfactorily obtained for both cases at both wavelengths, thus confirming the robustness of the pro- 

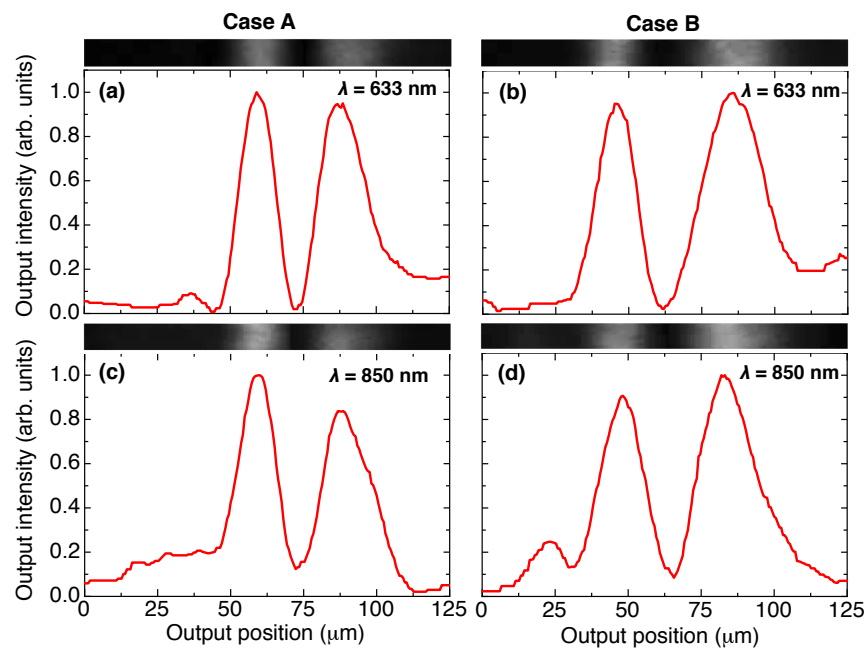

FIG. 7. Experimental output intensity distribution for the case of two-state STIRAP as probed for cases A and B at two different wavelengths. (a) and (b): probing at $\lambda=633$ $\mathrm{nm},(\mathrm{c})$ and (d): probing at $\lambda=850 \mathrm{~nm}$. Panels (a) and (c) correspond to case A, while panels (b) and (d) correspond to case B (see text).

cess. In absence of detuning the behavior would be expected to be highly dependent on wavelength, similar to the case of Fig. 4 for RAP. Therefore, the observed broadband behavior is a direct consequence of the waveguide detuning, which permits to place the experiments outside the region near the abscissa in Fig. 5(b), for which Rabi-like oscillations strongly dependent on wavelength would be expected.

\section{CONCLUSION}

We have discussed the role of waveguide detuning for the adiabatic light transfer among a pair of coupled waveguides in analogy with adiabatic quantum population dynamics processes. By the examples of the Rapid Adiabatic Passage and the two-state STIRAP processes the theoretical expectations have been successfully verified experimentally using photo-induced reconfigurable waveguides recorded in a photorefractive crystal. The corresponding structures act as broadband directional couplers and broadband beam splitters, respectively. The robustness and achromaticity brought about by the adiabatic evolution mediated by the detuning and the coupling strength have been confirmed. Generally, the combination of the effects of longitudinally varying coupling and longitudinally varying detuning permits to access a much wider range of possibilities for the adiabatic evolution of the light fields as compared to systems where only the coupling constant is modulated. As proposed recently for the case of three-waveguide couplers [38], this kind of concept can be extended to a larger number of waveguides, leading potentially to several new rich functionalities. The adiabatic approaches discussed here can be advantageously applied also to the case of nonclassical propagating light such as obtained from single photon sources. Also, by imposing an appropriate modulation of the mode propagation velocities, instead of standard dielectric waveguides also other types of guiding structures (e.g. photonic crystals or plasmonic waveguides) can be used in principle.

\section{ACKNOWLEDGMENTS}

This work has been supported by the Bulgaria-France bilateral program RILA and by the Region Lorraine.
[1] S. Longhi, Laser Photon. Rev. 3, 243 (2009).

[2] I. L. Garanovich, S. Longhi, A. A. Sukhorukov, and Y. S. Kivshar, Phys. Rep. 518, 1 (2012).

[3] U. Gaubatz, P. Rudecki, S. Schiemann, and K. Bergmann, J. Chem. Phys. 92, 5363 (1990).

[4] N. V. Vitanov, T. Halfmann, B. W. Shore, and K. Bergmann, Annu. Rev. Phys. Chem. 52, 763 (2001).

[5] N. V. Vitanov, A. A. Rangelov, B. W. Shore, and K. Bergmann, Rev. Modern Phys. (in press) (2017).

[6] E. Paspalakis, Optics Commun. 258, 30 (2006).

[7] S. Longhi, Phys. Rev. E 73, 026607 (2006).

[8] S. Longhi, G. Della Valle, M. Ornigotti, and P. Laporta, Phys. Rev. B 76, 201101 (2007).

[9] F. Dreisow, A. Szameit, M. Heinrich, S. Nolte, A. Tünnermann, M. Ornigotti, and S. Longhi, Appl. Phys. Lett. 95, 261102 (2009).

[10] G. Della Valle, M. Ornigotti, T. Toney Fernandez, P. LaPorta, and S. Longhi, Appl. Phys. Lett. 92, 011106 (2008).
[11] S.-Y. Tseng and M.-C. Wu, J. Lightwave Technol. 28, 3529 (2010).

[12] C. Ciret, V. Coda, A. A. Rangelov, D. N. Neshev, and G. Montemezzani, Phys. Rev. A 87, 013806 (2013).

[13] A. A. Rangelov and N. V. Vitanov, Phys. Rev. A 85, 055803 (2012).

[14] C. Ciret, V. Coda, A. A. Rangelov, D. N. Neshev, and G. Montemezzani, Opt. Lett. 37, 3789 (2012).

[15] W. Huang, A. A. Rangelov, and E. Kyoseva, Phys. Rev. A 90, 053837 (2014).

[16] A. P. Hope, T. G. Nguyen, A. Mitchell, and A. D. Greentree, J. Phys. B: At. Mol. Opt. Phys. 48, 055503 (2015).

[17] S.-Y. Tseng and M.-C. Wu, IEEE Photon. Technol. Lett. 22, 1211 (2010).

[18] Y. Lahini, F. Pozzi, M. Sorel, R. Morandotti, D. N. Christodoulides, and Y. Silberberg, Phys. Rev. Lett. 101, 193901 (2008).

[19] R. Menchon-Enrich, A. Llobera, J. Vila-Planas, V. J. Cadarso, J. Mompart, and V. Ahufinger, Light: Sci. 
Appl. 2, e90 (2013).

[20] R. Menchon-Enrich, A. Benseny, V. Ahufinger, A. D. Greentree, T. Busch, and J. Mompart, Rep. Prog. Phys. 79, 074401 (2016).

[21] A. Yariv, IEEE J. Quantum Electr. 9, 919 (1973).

[22] M. G. F. Wilson and G. A. Teh, Electron. Lett. 9, 453 (1973).

[23] R. B. Smith, J. Opt. Soc. Am. 66, 882 (1976).

[24] T. A. Ramadan, R. Scarmozzino, and R. M. Osgood Jr., J. Lightwave Technol. 16, 277 (1998).

[25] A. Abragam, The principles of nuclear magnetism (Clarendon, Oxford, 1961).

[26] E. B. Treacy, Phys. Lett. 27A, 421 (1968).

[27] M. M. T. Loy, Phys. Rev. Lett. 32, 814 (1974).

[28] S. M. Hamadani, A. T. Mattick, N. A. Kurnit, and A. Javan, Appl. Phys. Lett. 27, 21 (1975).

[29] S. Avrillier, J.-M. Raymond, C. J. Bordé, D. Bassi, and G. Scoles, Opt. Commun. 39, 311 (1981).

[30] J. P. C. Kroon, H. A. J. Senhorst, H. C. W. Beijerinck, B. J. Verhaar, and N. F. Verster, Phys. Rev. A 31, 3724 (1985).

[31] L. Allen and E. J. H., Optical resonance and two-level atoms (Wiley, New York, 1975).

[32] C. Leidenbaum, S. Stolte, and J. Reuss, Phys. Rep. 178, 1 (1989).
[33] N. V. Vitanov and B. W. Shore, Phys. Rev. A 73, 053402 (2006).

[34] L. P. Yatsenko, N. V. Vitanov, B. W. Shore, T. Rickes, and K. Bergmann, Opt. Commun. 204, 413 (2002).

[35] R. Yamazaki, K. I. Kanda, F. Inoue, K. Toyoda, and S. Urabe, Phys. Rev. A 78, 023808 (2008).

[36] M. Gorram, V. Coda, P. Thévenin, and G. Montemezzani, Appl. Phys. B 95, 565 (2009).

[37] C. Ciret, M. Alonzo, V. Coda, A. A. Rangelov, and G. Montemezzani, Phys. Rev. A 88, 013840 (2013).

[38] H. S. Hristova, A. A. Rangelov, G. Montemezzani, and N. V. Vitanov, Phys. Rev. A 93, 033802 (2016).

[39] B. Shore, The theory of coherent atomic excitation (John Wiley \& Sons, New York, 1990).

[40] P. Dittrich, G. Montemezzani, P. Bernasconi, and P. Günter, Opt. Lett. 24, 1508 (1999).

[41] G. Montemezzani, M. Gorram, N. Fressengeas, F. Juvalta, M. Jazbinsek, and P. Günter, Opt. Express 16, 16646 (2008).

[42] R. P. Feynman, F. L. Vernon Jr., and R. W. Hellwarth, J. Appl. Phys. 28, 49 (1957).

[43] J. Van Roey, J. van der Donk, and P. E. Lagasse, J. Opt. Soc. Am. 71, 803 (1981).

[44] K. Kawano and T. Kitoh, Introduction to optical waveguide analysis solving Maxwell's equations and the Schrödinger equation (John Wiley \& Sons, New York, 2001). 